\title{
EMPREGO DE UM MODELO DE CRESCIMENTO E PRODUÇÃO EM POVOAMENTOS DESBASTADOS DE EUCALIPTO ${ }^{1}$
}

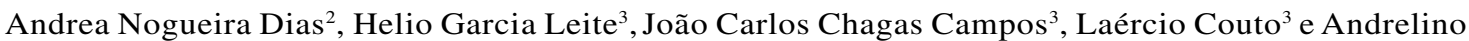 \\ Ferreira de Carvalho ${ }^{4}$
}

\begin{abstract}
RESUMO - O presente estudo teve como objetivo avaliar a aplicabilidade do modelo de Clutter em povoamentos desbastados de eucalipto. Esta pesquisa foi desenvolvida com dados da empresa Copener Florestal Ltda., atualmente Bahia Pulp, no Município de Alagoinhas, BA. Os dados utilizados fazem parte de um experimento de desbaste implantado em povoamentos de eucalipto, empregando-se o esquema de blocos casualizados constituídos de quatro tratamentos e repetidos em três locais diferentes, perfazendo 48 parcelas com área média de 2.600 $\mathrm{m}^{2}$ cada uma. As parcelas foram medidas aos 27, 40, 50, 58, 61 e 76 meses de idade, e aos 58 meses realizouse o desbaste. Para ajuste do modelo, foi empregado o sistema de equações simultâneas, que utiliza informações do povoamento referentes a área basal, volume, idade e índice de local. A partir do ajuste do modelo, elaborouse uma tabela de produção de densidade variável e determinou-se a idade técnica de corte em diferentes capacidades produtivas. Realizaram-se simulações na tabela de produção de regimes de desbaste. Concluiu-se que o modelo de Clutter foi eficiente para predizer a produção atual e futura dos povoamentos desbastados de eucalipto, mostrando-se útil na análise de alternativas de manejo, como a determinação da idade técnica de desbaste, de diferentes índices de local e regimes de desbaste.
\end{abstract}

Palavras-chave: Modelo de crescimento e produção, desbaste, eucalipto e idade técnica de corte.

\section{THE USE OF A GROWTH AND YIELD MODEL IN THINNED EUCALYPT STANDS}

\begin{abstract}
The objective of the present study was to evaluate the applicability of the Clutter model in thinned eucalypt stands. The study was developed with data from the Copener Florestal, Bahia Pulp Enterprise, located in the municipality of Alagoinhas, Bahia, Brazil. Data came from a thinning experiment established in eucalypt stands using a randomized block design with four treatments and three repetitions, each one in a different place, totaling 48 plots with a mean area of 2,600 $\mathrm{m}^{2}$ each. The plots were measured at 27, 40, 50, 58, 61 and 76 months of age, and the thinning was carried out at 58 months. For model adjustment, a system of simultaneous equations was used with stand information such as basal area, volume, age and site index. From the model adjustment, a yield table with variable density was built, and the technical cutting age was determined for various thinning intensities. Simulations were carried out with the yield table. It was concluded that the Clutter model was efficient to predict the present and future yields of the thinned eucalypt stands, showing its usefulness in the analysis of alternative managements, such as the technical cutting age determination for various site indexes and thinning intensities.
\end{abstract}

Keywords: growth and yield model, thinning, eucalypt and technical cutting age.

\footnotetext{
${ }^{1}$ Recebido em 04.04.2003 e aceito para publicação em 10.08.2005.

${ }^{2}$ Departamento de Engenharia Florestal da UNICENTRO, 84500-000 Irati -PR. <andias@ irati.unicentro.br>.

${ }^{3}$ Departamento de Engenharia Florestal da UFV, 36570-000 Viçosa-MG. <hgleite@.ufv.br>, <lcouto@ufv.br>.

${ }^{4}$ COPENER FLORESTAL, BA.
} 


\section{INTRODUÇÃO}

Nas últimas décadas, as empresas florestais têm alterado o manejo tradicional dos povoamentos de eucaliptos, com rotação em torno de sete anos, às vezes com a condução de uma, duas e até três talhadias, por ciclos de corte maiores, com aplicação de desbaste e de desrama artificial.

A aplicação do desbaste pode resultar na produção de árvores de grande porte, se houver melhor distribuição dos fatores de crescimento, como água, luz e nutrientes para as árvores, previamente selecionadas, com a garantia de aumento na qualidade da madeira, o que possivelmente irá agregar valor a esta.

Em países como África do Sul, Zimbabwe, Austrália, EUA e Costa Rica existem pesquisas e áreas comerciais de Eucalyptus conduzidos através de regimes de desbaste, com objetivos principais de obtenção de madeira para fins estruturais e serraria (STAPE e MARTINI, 1991). No Brasil, a aplicação operacional de desbaste em povoamentos de eucalipto ainda não é comum, muitas vezes havendo necessidade de definir quanto da floresta será submetido a desbaste, a idade de executá-lo e sua intensidade.

A carência de experimentos nesta área está relacionada ao fato de poucos povoamentos de eucalipto terem sido conduzidos com a aplicação de desbaste (NOGUEIRA et. al., 2001).

Pienaar e Shiver (1984) já mencionavam que em um povoamento florestal a taxa de crescimento de árvores individuais depende da quantidade de espaço de crescimento disponível e que, por essa razão, muitos estudos estavam sendo realizados para prover informações sobre estratégias de desbaste, cabendo citar Clutter e Jones (1980), Matney e Sullivan (1982) e Bailey e Ware (1983).

Uma alternativa para quantificar o efeito do desbaste sobre a produção de madeira consiste no emprego de modelos de crescimento e produção. É oportuno diferenciar os tipos de modelo que podem ser utilizados. Sistemas de equações que compreendem estimativas de volume por unidade de área são definidos como sistemas de predição explícita, ou modelos do tipo povoamento total. No entanto, sistemas que requerem informações básicas da produção e da estrutura do povoamento são chamados de sistemas de predição implícita, ou modelos de distribuição de diâmetros.
Outro tipo de modelo existente que utiliza informações da árvore individual como unidade básica para a predição, é conhecido como modelos de árvores individuais (CLUTTER et. al., 1983).

No Brasil, persiste o uso de modelos em nível de povoamento, ou seja, em que a produção em volume e área basal é projetada com base nas características do povoamento como idade, área basal e índice de local. Esse tipo de modelo, especialmente o de Clutter (1963), tem sido utilizado com freqüência para o manejo de plantações não desbastadas de eucalipto.

Ao analisar os artigos publicados na literatura, observou-se que existem poucos trabalhos utilizando modelos de crescimento e produção desenvolvidos para povoamentos de eucalipto que foram efetivamente submetidos a desbaste. Na maioria das vezes, tratase de estudos com dados de povoamentos não desbastados, em que se utilizam os modelos para se fazerem simulações. Algumas das publicações sobre modelagem utilizando dados de florestas submetidas a desbaste foram conduzidos usando os dados reportados em O'Connor em 1935 e estabelecidos na África do Sul por Craib, em 1939 (HILEY, 1959).

Dessa forma, realizou-se este estudo com o objetivo de avaliar a eficiência do modelo de Clutter para predizer o crescimento e a produção em volume e área basal em povoamentos de eucalipto submetidos a desbaste.

\section{MATERIAL E MÉTODOS}

\subsection{Modelo Utilizado e Fonte de Dados}

Foram utilizados dados provenientes de uma rede de parcelas de um experimento estabelecido em plantações do híbrido Eucalyptus grandis X Eucalyptus urophylla, pertencentes à empresa COPENER FLORESTAL Ltda., atualmente Bahia Pulp, localizada no Município de Alagoinhas, BA.

O delineamento utilizado foi o de blocos casualizados em três instalações com dois blocos em cada uma delas, com duas repetições por bloco. Cada bloco foi constituído por quatro tratamentos, perfazendo 48 parcelas, com cerca de $2.600 \mathrm{~m}^{2}$ de área útil cada uma.

Os tratamentos aplicados foram diferenciados em $\%$ de área basal retirada por hectare: $\mathrm{T} 1=20 \% \mathrm{de}$ desbaste, $\mathrm{T} 2=35 \%$ de desbaste, $\mathrm{T} 3=50 \%$ de desbaste, e T $4=35 \%$ de desbaste + desrama. Os tratamentos foram efetivamente aplicados na idade de 58 meses, sendo o desbaste do tipo misto (seletivo e sistemático). 
O modelo do tipo povoamento total, selecionado para este estudo, foi proposto por Clutter (1963). Tratase de um modelo de propriedade compatível entre o crescimento e a produção.

Para ajustar o modelo de Clutter foi necessário obter as informações de volume, área basal, índice de local e idade do povoamento em cada medição ( 27 , $40,50,58,61$ e 76 meses). O volume observado foi obtido a partir da equação de volume de árvores individuais ajustado por Dias (2000). Os valores de índices de local foram obtidos a partir do método da curva-guia, apresentado por esse autor. Para determinação da produção total em volume e área basal, as variáveis foram totalizadas por unidade de área (hectare) em cada parcela.

O modelo utilizado foi o modelo de equações simultâneas de Clutter, assim descrito:

$$
\begin{aligned}
& \operatorname{LnV}_{2 \mathrm{i}}=\beta_{1}+\beta_{2} / \mathrm{I}_{2 \mathrm{i}}+\beta_{3} \mathrm{~S}_{\mathrm{i}}+\beta_{4} \operatorname{LnB}_{2 \mathrm{i}}+\mathrm{u}_{\mathrm{i}} \\
& \mathrm{LnB}_{2 \mathrm{i}}=\operatorname{LnB}_{1}\left(\frac{\mathrm{I}_{1 \mathrm{i}}}{\mathrm{I}_{2 \mathrm{i}}}\right)+\alpha_{0}\left(1-\frac{\mathrm{I}_{1 \mathrm{i}}}{\mathrm{I}_{2 \mathrm{i}}}\right)+\mathrm{u}_{\mathrm{i}}
\end{aligned}
$$

em que:

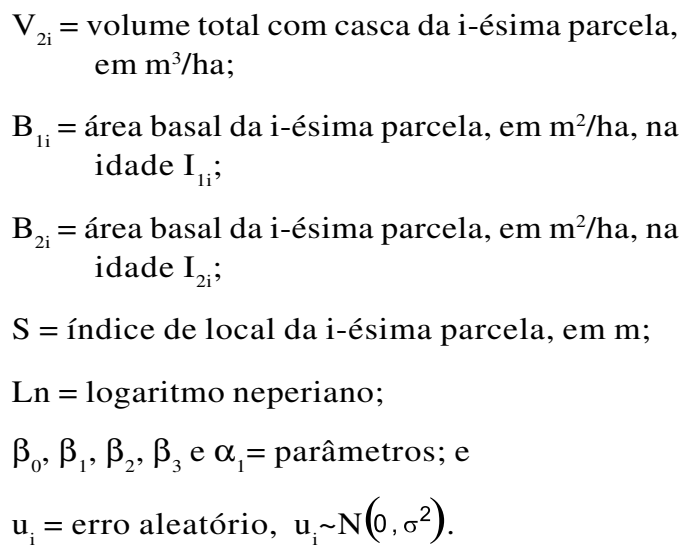

O sistema de equações simultâneas, desenvolvido por Clutter em 1963, tem como variáveis endógenas o volume e área basal e como variáveis exógenas, a idade, o índice de local e a área basal inicial.

A partir dos resultados da análise gráfica de resíduos e das medidas de precisão, optou-se por retirar a variável "S" no modelo (2), devido à ocorrência de valor negativo para o parâmetro associado ao índice de local na equação de área basal, gerando inconsistências do ponto de vista biológico. Resultados semelhantes foram encontrados em Gastelurrutia e Gonzáles (2001). ValdezLazalde e Lynch (2000) optaram também por retirar essa variável, por apresentar valor não-significativo estatisticamente.

\subsection{Avaliação do modelo}

Para avaliar o modelo ajustado e verificar a existência ou não de tendenciosidade nas estimativas, utilizaramse as seguintes estatísticas: coeficiente de determinação $\left(\mathrm{R}^{2}\right)$, significância dos coeficientes avaliada pelo teste de análise gráfica de resíduos. Além disso, a comparação entre os valores observados e estimados nos modelos (1) e (2) envolveu uma análise do erro médio porcentual da estimativa, a correlação e a estatística erro de tendência.

Sendo consideradas como precisas, segundo testes aferidos por Guimarães (1994), para estudo desta natureza, as estimativas de volume e área basal resultaram em: $\mathrm{r}_{\mathrm{VeV}} \geq 0,80$, Bias $<20 \%$ e $\overline{\mathrm{e}} \%<10$, em que:

$$
\begin{aligned}
& \mathrm{r}_{\mathrm{v}} \mathrm{v}_{\mathrm{o}}=\frac{\operatorname{Cô}\left(\mathrm{V}_{\mathrm{e}}, \mathrm{V}_{\mathrm{o}}\right)}{\sqrt{\mathrm{V}\left(\mathrm{V}_{\mathrm{e}}\right) \mathrm{V}\left(\mathrm{V}_{\mathrm{o}}\right)}}=\text {, erro de tendência }=100 \sum \frac{\left(\mathrm{V}_{\mathrm{e}}-\mathrm{V}_{\mathrm{o}}\right)}{\mathrm{n}}, \\
& \overline{\mathrm{e}} \%= \frac{\sum\left(\frac{\mathrm{V}_{\mathrm{o}}-\mathrm{V}_{\mathrm{e}}}{\mathrm{V}_{\mathrm{o}}}\right)}{\mathrm{n}} * 100 ; \\
& \mathrm{V}_{0}=\text { volume observado }\left(\mathrm{m}^{3} / \mathrm{ha}\right) ; \mathrm{e} \\
& \mathrm{Ve}=\text { volume estimado }\left(\mathrm{m}^{3} / \mathrm{ha}\right) .
\end{aligned}
$$

Para demonstrar a aplicabilidade do modelo para predição do sistema de equações, elaborou-se uma tabela de produção de densidade variável (TPDV). A partir dessa tabela, pode-se analisar a consistência das estimativas em relação aos efeitos da área basal inicial e do índice de local sobre a idade técnica de corte (ITC).

\section{RESULTADOS E DISCUSSÃO}

\subsection{Avaliação do modelo}

O método utilizado no ajuste do modelo de Clutter foi o dos mínimos quadrados em dois estágios. As equações resultantes, bem como as medidas de precisão, são apresentadas a seguir:

1) Produção futura em volume:

$$
\begin{aligned}
& \operatorname{LnV}_{2}=2,441896-24,23312 / I_{2}+0,032370 \mathrm{~S}+0,726114 \mathrm{LnB}_{2} \\
& \begin{array}{lllll}
(15,790) & (-13,445) & (4,9352) & (17,771) & \text { estatística } t
\end{array} \\
& (0,000) \quad(0,000) \quad(0,000) \quad(0,000) \quad \mathrm{p} \text {-valor } \\
& \mathrm{R}^{2}=0,73 \quad \text { Erro de tendência }=-19,3825 \quad \overline{\mathrm{e}} \%=-0,5242
\end{aligned}
$$


2) Produção futura em área basal:

$$
\begin{aligned}
\operatorname{LnB}_{2}=\operatorname{LnB}_{1}\left(\frac{I_{1}}{I_{2}}\right) & +3,2865\left(1-\frac{I_{1}}{I_{2}}\right) \\
& (160,359) \text { estatística } t \\
& (0,000) \quad p \text { - valor }
\end{aligned}
$$

$\mathrm{R}^{2}=0,88 \quad$ Erro de tendência $=-0,677 \quad \overline{\mathrm{e}} \%=-0,2041$

Verificou-se que os resultados estão adequados, ou seja, de acordo com o p-valor, todos os coeficientes estimados dos modelos (1) e (2) são significativos em nível de $1 \%$ de probabilidade. Além disso, as equações apresentam coeficientes de determinação satisfatórios,

Na comparação entre os valores observados e estimados para os modelos (1) e (2), observou-se erro médio porcentual menor que 10 nos dois modelos, e a correlação entre os valores observados e estimados tanto para volume quanto para área basal foi $>0,80$ e a estatística erro de tendência para os modelos (1) e $(2),<20$. As estimativas de produção em volume $\left(\mathrm{m}^{3} /\right.$ ha) e área basal ( $\left.\mathrm{m}^{2} / \mathrm{ha}\right)$ foram consideradas precisas.
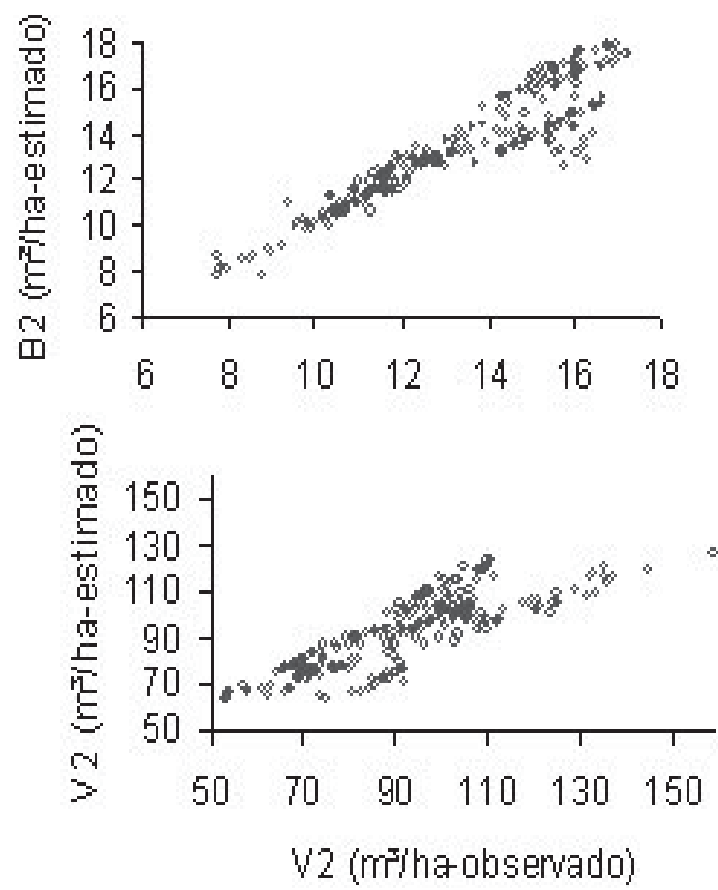
indicando bom ajustamento do modelo.

Em seguida, foram analisadas as distribuições dos resíduos geradas pela aplicação do modelo (Figura 1). Dessa análise, observou-se que houve uma leve tendência nas extremidades da projeção, que são derivadas das mudanças ocorridas no povoamento após a aplicação do desbaste, no entanto os resultados foram considerados aceitáveis.

\subsection{Construção das Tabelas de Produção de Densidade Variável (TPDV)}

Geralmente, a idade técnica de corte é utilizada quando o objetivo é a maximização da produtividade em volume. Dessa forma, corta-se o povoamento na idade de máximo incremento médio anual (IMA) (LEUSCHNER, 1990). Essa idade corresponde à idade em que o incremento médio anual (IMA) se iguala ao incremento corrente anual (ICA). Segundo pressupostos teóricos, a ITC tende a ser atingida mais cedo em locais de maior capacidade produtiva, porque nesses locais as árvores atingem a estagnação do crescimento mais precocemente (LEITE, 2001).
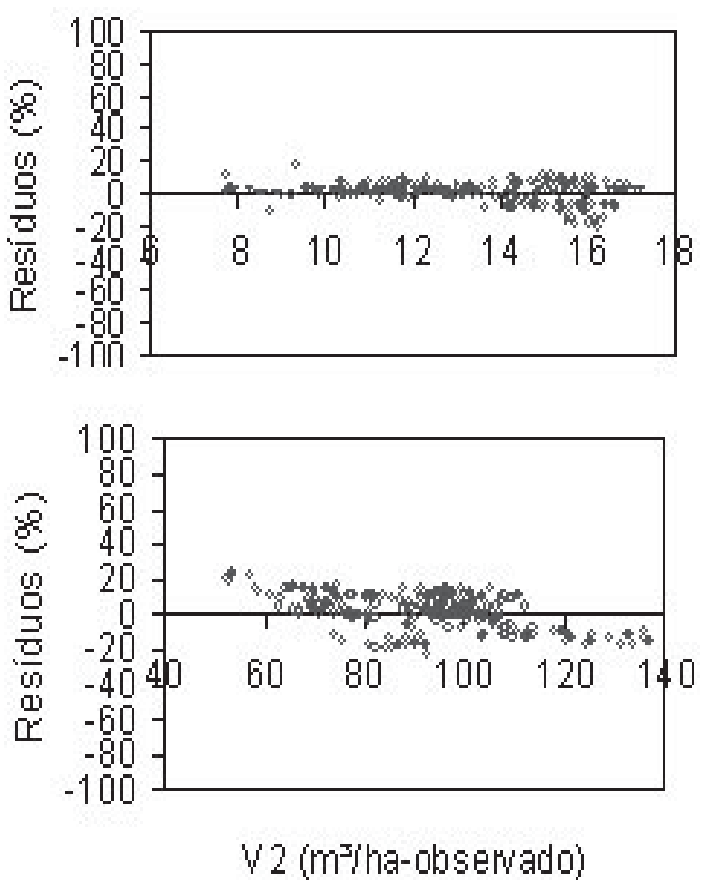

Figura 1 - Análises gráficas elaboradas para verificação de tendenciosidade das estimativas de volume e área basal. Figure 1 -Graphical analyses of residuals to verify the bias of volume and basal area estimation.

R. Árvore, Viçosa-MG, v.29, n.5, p.731-739, 2005 
Uma vez devidamente avaliadas, as equações foram utilizadas para estimar as produções presente e futura em volume e área basal e para calcular os incrementos médios mensais (IMM). Tais informações foram utilizadas para a construção da tabela de produção de densidade variável (Quadro 1).

Como a equação de predição em área basal requer um valor de área basal inicial, usou-se na construção da tabela para diferentes índices de local o seguinte critério: para o índice de local 17, utilizou-se a área basal inicial média observada aos 27 meses - desvio $\left(\mathrm{AB}_{27}-\hat{\sigma}_{27}\right)$; para o índice de local 21 , a área basal inicial média observada aos 27 meses $\left(A \bar{B}_{27}\right)$; e para o índice de local 25, a área basal inicial média, verificada aos 27 meses + desvio $\left(\mathrm{AB}_{27}+\hat{\sigma}_{27}\right)$.
A partir da tabela de produção de densidade variável foi possível determinar a idade de máximo incremento médio mensal correspondente à idade técnica de corte (ITC) para cada índice de local. Observou-se na TPDV que a ITC diminuiu à medida que se aumentou o índice de local, ou seja, para o índice de local 25 a ITC foi de 43 meses, enquanto para o índice de local 21 foi de 44 meses e para 17, de 46 meses. Esses resultados confirmam a teoria de que local de melhor capacidade produtiva tende a proporcionar uma antecipação da idade de estagnação do crescimento dos povoamentos.

Para melhor compreensão do efeito do índice de local sobre a idade técnica de corte (ITC), ou seja, idade em que a curva de incremento corrente mensal (ICM) cruza com a curva do incremento médio mensal (IMM), elaborou-se a Figura 2.

Quadro 1 - Tabela de produção de densidade variável para o híbrido Eucalyptus grandis X E.urophylla, sem simulação de desbaste

Table 1 - Variable density yield table for the Eucalyptus grandis X E. urophylla hybrid, without thinning simulation

\begin{tabular}{|c|c|c|c|c|c|c|c|c|c|}
\hline \multirow[b]{3}{*}{$\begin{array}{l}\text { Idade } \\
\text { (meses) }\end{array}$} & \multicolumn{9}{|c|}{ Índice de Local } \\
\hline & \multicolumn{3}{|c|}{17} & \multicolumn{3}{|c|}{21} & \multicolumn{3}{|c|}{25} \\
\hline & $\begin{array}{c}\text { Á. Basal } \\
\left(\mathrm{m}^{2} / \mathrm{ha}\right)\end{array}$ & $\begin{array}{l}\text { Volume } \\
\left(\mathrm{m}^{3} / \mathrm{ha}\right)\end{array}$ & IMM & $\begin{array}{l}\text { Á. Basal } \\
\left(\mathrm{m}^{2} / \mathrm{ha}\right)\end{array}$ & $\begin{array}{l}\text { Volume } \\
\left(\mathrm{m}^{3} / \mathrm{ha}\right)\end{array}$ & IMM & $\begin{array}{l}\text { Á. Basal } \\
\left(\mathrm{m}^{2} / \mathrm{ha}\right)\end{array}$ & $\begin{array}{l}\text { Volume } \\
\left(\mathrm{m}^{3} / \mathrm{ha}\right)\end{array}$ & IMM \\
\hline 24 & 7,50 & 31,36 & 1,307 & 8,50 & 39,09 & 1,629 & 9,00 & 46,38 & 1,932 \\
\hline 26 & 8,27 & 36,39 & 1,400 & 9,28 & 45,04 & 1,732 & 9,79 & 53,27 & 2,049 \\
\hline 28 & 8,99 & 41,33 & 1,476 & 10,01 & 50,86 & 1,816 & 10,52 & 59,99 & 2,142 \\
\hline 30 & 9,67 & 46,16 & 1,539 & 10,69 & 56,5 & 1,883 & 11,19 & 66,49 & 2,216 \\
\hline 32 & 10,31 & 50,85 & 1,589 & 11,32 & 61,96 & 1,936 & 11,82 & 72,75 & 2,273 \\
\hline 34 & 10,9 & 55,37 & 1,629 & 11,91 & 67,2 & 1,977 & 12,4 & 78,77 & 2,317 \\
\hline 36 & 11,46 & 59,73 & 1,659 & 12,46 & 72,24 & 2,007 & 12,94 & 84,53 & 2,348 \\
\hline 38 & 11,98 & 63,93 & 1,682 & 12,97 & 77,06 & 2,028 & 13,44 & 90,04 & 2,370 \\
\hline 40 & 12,47 & 67,95 & 1,699 & 13,45 & 81,68 & 2,042 & 13,91 & 95,31 & 2,383 \\
\hline 42 & 12,93 & 71,81 & 1,710 & 13,89 & 86,09 & 2,050 & 14,35 & 100,34 & 2,389 \\
\hline 43 & 13,15 & 73,67 & 1,713 & 14,11 & 88,22 & 2,051 & 14,56 & 102,77 & 2,390 \\
\hline 44 & 13,37 & 75,50 & 1,716 & 14,31 & 90,31 & 2,053 & 14,77 & 105,15 & 2,389 \\
\hline 46 & 13,78 & 79,05 & 1,718 & 14,71 & 94,34 & 2,051 & 15,15 & 109,74 & 2,386 \\
\hline 48 & 14,16 & 82,44 & 1,717 & 15,08 & 98,2 & 2,046 & 15,52 & 114,12 & 2,377 \\
\hline 50 & 14,53 & 85,69 & 1,714 & 15,43 & 101,88 & 2,038 & 15,86 & 118,3 & 2,366 \\
\hline 52 & 14,87 & 88,80 & 1,708 & 15,76 & 105,41 & 2,027 & 16,18 & 122,3 & 2,352 \\
\hline 54 & 15,2 & 91,79 & 1,700 & 16,07 & 108,78 & 2,014 & 16,48 & 126,12 & 2,336 \\
\hline 56 & 15,51 & 94,65 & 1,690 & 16,37 & 112,01 & 2,000 & 16,77 & 129,78 & 2,317 \\
\hline 58 & 15,81 & 97,39 & 1,679 & 16,65 & 115,1 & 1,984 & 17,04 & 133,28 & 2,298 \\
\hline 60 & 16,09 & 100,02 & 1,667 & 16,91 & 118,06 & 1,968 & 17,3 & 136,63 & 2,277 \\
\hline 62 & 16,35 & 102,55 & 1,654 & 17,16 & 120,9 & 1,950 & 17,55 & 139,84 & 2,256 \\
\hline 64 & 16,6 & 104,97 & 1,640 & 17,40 & 123,63 & 1,932 & 17,78 & 142,92 & 2,233 \\
\hline 66 & 16,85 & 107,30 & 1,626 & 17,63 & 126,24 & 1,913 & 18,00 & 145,88 & 2,210 \\
\hline 68 & 17,08 & 109,6 & 1,611 & 17,85 & 128,75 & 1,893 & 18,21 & 148,71 & 2,187 \\
\hline 70 & 17,3 & 111,7 & 1,596 & 18,06 & 131,17 & 1,874 & 18,41 & 151,44 & 2,163 \\
\hline 72 & 17,51 & 113,78 & 1,580 & 18,25 & 133,49 & 1,854 & 18,61 & 154,06 & 2,140 \\
\hline 74 & 17,71 & 115,78 & 1,565 & 18,44 & 135,72 & 1,834 & 18,79 & 156,58 & 2,116 \\
\hline 76 & 17,9 & 117,7 & 1,549 & 18,63 & 137,87 & 1,814 & 18,96 & 159,00 & 2,092 \\
\hline 78 & 18,09 & 119,56 & 1,533 & 18,80 & 139,95 & 1,794 & 19,13 & 161,34 & 2,068 \\
\hline 80 & 18,27 & 121,35 & 1,517 & 18,97 & 141,94 & 1,774 & 19,29 & 163,59 & 2,045 \\
\hline 82 & 18,44 & 123,08 & 1,501 & 19,13 & 143,87 & 1,755 & 19,45 & 165,76 & 2,021 \\
\hline
\end{tabular}

Idade técnica de corte. 


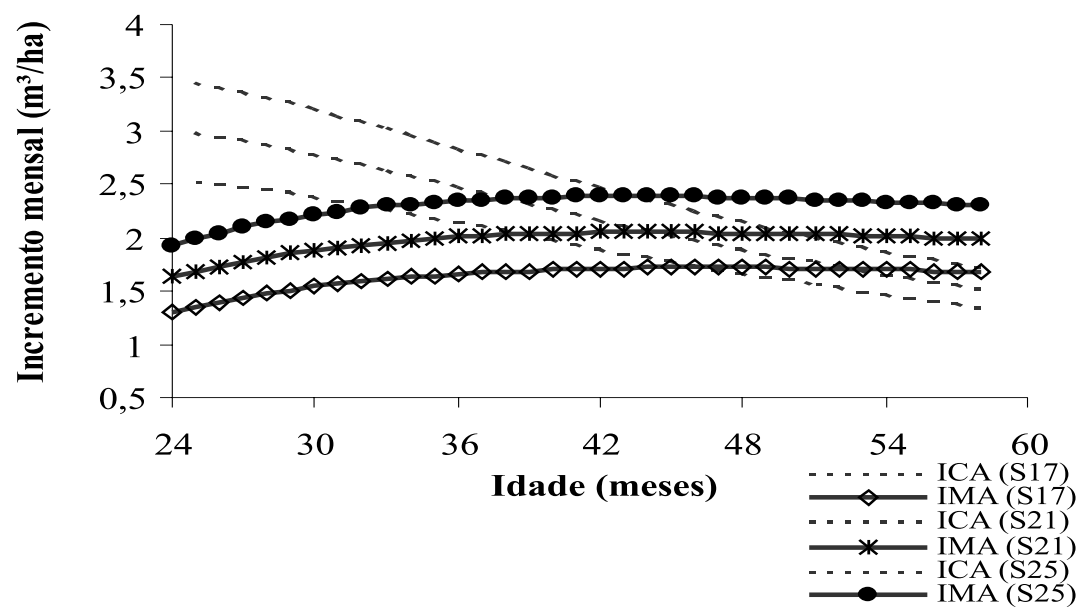

Figura 2 - Curvas de incrementos correntes mensais e incrementos médios mensais dos índices de local 17, 21 e 25. Figure 2 - Curves of monthly current increments and monthly mean increments for the site indexes 17, 21, and 25.

\subsection{Simulação de desbaste}

Modelos de crescimento e produção podem ser utilizados para analisar alternativas silviculturais, por exemplo simulações de diferentes intensidades de desbaste realizado no povoamento. Neste estudo, realizaram-se simulações de desbaste na TPDV, com o objetivo de obter o volume desbastado e o volume do corte final para cada situação. As simulações se referem a diferentes idades de desbaste, intensidades e índices de local.

Quadro 2 - TPDV considerando a ocorrência de desbaste, com redução de 35\% da área basal para o híbrido Eucalyptus grandis $X$ E.urophylla

Table 2 - TPDV considering thinning occurrencewith $35 \%$ reduction of basal area for the Eucalyptus grandis X E. urophylla hybrid

\begin{tabular}{|c|c|c|c|c|c|c|c|c|c|}
\hline \multirow[b]{3}{*}{$\begin{array}{l}\text { Idade } \\
\text { (meses) }\end{array}$} & \multicolumn{9}{|c|}{ Índice de Local } \\
\hline & \multicolumn{3}{|c|}{17} & \multicolumn{3}{|c|}{21} & \multicolumn{3}{|c|}{25} \\
\hline & $\begin{array}{l}\text { Á. Basal } \\
\left(\mathrm{m}^{2} / \mathrm{ha}\right)\end{array}$ & $\begin{array}{l}\text { Volume } \\
\left(\mathrm{m}^{3} / \mathrm{ha}\right)\end{array}$ & IMM & $\begin{array}{c}\text { Á. Basal } \\
\left(\mathrm{m}^{2} / \mathrm{ha}\right)\end{array}$ & $\begin{array}{l}\text { Volume } \\
\left(\mathrm{m}^{3} / \mathrm{ha}\right)\end{array}$ & IMM & $\begin{array}{c}\text { Á. Basal } \\
\left(\mathrm{m}^{2} / \mathrm{ha}\right)\end{array}$ & $\begin{array}{l}\text { Volume } \\
\left(\mathrm{m}^{3} / \mathrm{ha}\right)\end{array}$ & IMM \\
\hline 24 & 7,50 & 31,36 & 1,307 & 8,5 & 39,09 & 1,629 & 9,00 & 46,38 & 1,933 \\
\hline 30 & 9,67 & 46,16 & 1,539 & 10,69 & 56,5 & 1,883 & 11,19 & 66,49 & 2,216 \\
\hline 36 & 11,46 & 59,73 & 1,659 & 12,46 & 72,24 & 2,007 & 12,94 & 84,53 & 2,348 \\
\hline $40^{*}$ & 12,47 & 67,95 & 1,699 & 13,45 & 81,67 & 2,042 & 9,04 & 69,71 & 1,743 \\
\hline $41^{\text {*** }}$ & 12,71 & 69,90 & 1,705 & 8,89 & 61,37 & 1,497 & 9,29 & 72,12 & 1,759 \\
\hline 42 & 12,93 & 71,81 & 1,710 & 9,12 & 63,44 & 1,510 & 9,52 & 74,49 & 1,774 \\
\hline $43^{\text {*k*k }}$ & 8,55 & 53,89 & 1,253 & 9,58 & 67,48 & 1,534 & 9,76 & 76,83 & 1,787 \\
\hline 48 & 9,63 & 62,29 & 1,298 & 10,84 & 78,83 & 1,577 & 10,84 & 87,93 & 1,832 \\
\hline 54 & 10,79 & 71,55 & 1,325 & 11,94 & 89,08 & 1,591 & 11,98 & 100,04 & 1,852 \\
\hline 56 & 11,14 & 74,44 & 1,329 & 12,28 & 92,26 & 1,591 & 12,33 & 103,79 & 1,853 \\
\hline 57 & 11,31 & 75,84 & 1,331 & 12,11 & 90,69 & 1,591 & 12,5 & 105,62 & 1,853 \\
\hline 58 & 11,48 & 77,23 & 1,332 & 12,6 & 95,34 & 1,589 & 12,66 & 107,42 & 1,852 \\
\hline 60 & 11,81 & 79,93 & 1,332 & 12,91 & 98,31 & 1,586 & 12,98 & 110,91 & 1,849 \\
\hline 66 & 12,72 & 87,52 & 1,326 & 13,77 & 106,62 & 1,568 & 13,86 & 120,68 & 1,828 \\
\hline 72 & 13,54 & 94,39 & 1,311 & 14,53 & 114,12 & 1,542 & 14,64 & 129,48 & 1,798 \\
\hline 78 & 14,26 & 100,62 & 1,290 & 15,21 & 120,91 & 1,511 & 15,34 & 137,42 & 1,762 \\
\hline 82 & 14,71 & 104,46 & 1,274 & 15,42 & 123,04 & 1,500 & 15,76 & 142,30 & 1,735 \\
\hline
\end{tabular}

Idade técnica de corte.

"Idade de desbaste dos índices de local $25,{ }^{* *}$ Idade de desbaste dos índices de local 21 e ${ }^{* * *}$ Idade de desbaste do índice de local 17.

R. Árvore, Viçosa-MG, v.29, n.5, p.731-739, 2005 
Emprego de um modelo de crescimento e produção ...

Quadro 3 - TPDV considerando a ocorrência de desbaste, com redução de 50\% da área basal, do híbrido Eucalyptus grandis $X$ E.urophylla, no município de Alagoinhas, Bahia

Table 3 - TPDV considering thinning occurrence, with 50\% reduction of basal área for the Eucalyptus grandis X E. urophylla hybrid, in the municipality of Alagoinhas, Bahia

\begin{tabular}{|c|c|c|c|c|c|c|c|c|c|}
\hline \multirow[b]{3}{*}{$\begin{array}{l}\text { Idade } \\
\text { (meses) }\end{array}$} & \multicolumn{9}{|c|}{ Índice de Local } \\
\hline & \multicolumn{3}{|c|}{17} & \multicolumn{3}{|c|}{21} & \multicolumn{3}{|c|}{25} \\
\hline & $\begin{array}{c}\text { Á. Basal } \\
\left(\mathrm{m}^{2} / \mathrm{ha}\right)\end{array}$ & $\begin{array}{l}\text { Volume } \\
\left(\mathrm{m}^{3} / \mathrm{ha}\right)\end{array}$ & IMM & $\begin{array}{c}\text { Á. Basal } \\
\left(\mathrm{m}^{2} / \mathrm{ha}\right)\end{array}$ & $\begin{array}{l}\text { Volume } \\
\left(\mathrm{m}^{3} / \mathrm{ha}\right)\end{array}$ & IMM & $\begin{array}{c}\text { Á. Basal } \\
\left(\mathrm{m}^{2} / \mathrm{ha}\right)\end{array}$ & $\begin{array}{l}\text { Volume } \\
\left(\mathrm{m}^{3} / \mathrm{ha}\right)\end{array}$ & IMM \\
\hline 24 & 7,5 & 31,36 & 1,307 & 8,5 & 39,09 & 1,629 & 9,00 & 46,38 & 1,933 \\
\hline 30 & 9,67 & 46,16 & 1,539 & 10,69 & 56,5 & 1,883 & 11,19 & 66,49 & 2,216 \\
\hline 36 & 11,46 & 59,73 & 1,659 & 12,46 & 72,24 & 2,007 & 12,94 & 84,53 & 2,348 \\
\hline $40^{*}$ & 12,47 & 67,95 & 1,699 & 13,45 & 81,67 & 2,042 & 6,96 & 57,62 & 1,441 \\
\hline $41^{* *}$ & 12,71 & 69,9 & 1,705 & 6,84 & 50,72 & 1,237 & 7,19 & 59,89 & 1,461 \\
\hline 42 & 12,93 & 71,81 & 1,710 & 7,06 & 52,67 & 1,254 & 7,42 & 62,13 & 1,479 \\
\hline $43^{* * * *}$ & 6,58 & 44,54 & 1,036 & 7,28 & 54,6 & 1,270 & 7,64 & 64,35 & 1,497 \\
\hline 48 & 7,61 & 52,52 & 1,094 & 8,34 & 63,88 & 1,331 & 8,71 & 75,02 & 1,563 \\
\hline 54 & 8,75 & 61,48 & 1,139 & 9,49 & 74,23 & 1,375 & 9,86 & 86,87 & 1,609 \\
\hline 60 & 9,79 & 69,73 & 1,162 & 10,53 & 83,7 & 1,395 & 10,9 & 97,68 & 1,628 \\
\hline 63 & 10,27 & 73,6 & 1,168 & 11,01 & 88,12 & 1,399 & 11,38 & 102,72 & 1,630 \\
\hline 64 & 10,42 & 74,85 & 1,170 & 11,16 & 89,55 & 1,399 & 11,53 & 104,35 & 1,630 \\
\hline 65 & 10,57 & 76,09 & 1,170 & 11,31 & 90,96 & 1,399 & 11,68 & 105,95 & 1,630 \\
\hline 66 & 10,72 & 77,3 & 1,171 & 11,46 & 92,34 & 1,399 & 11,83 & 107,52 & 1,629 \\
\hline 68 & 11,02 & 79,68 & 1,171 & 11,75 & 95,05 & 1,398 & 12,11 & 110,6 & 1,626 \\
\hline 72 & 11,57 & 84,24 & 1,170 & 12,3 & 100,22 & 1,392 & 12,39 & 113,58 & 1,623 \\
\hline 78 & 12,34 & 90,59 & 1,161 & 13,06 & 107,41 & 1,377 & 13,41 & 124,63 & 1,598 \\
\hline 82 & 12,82 & 94,53 & 1,153 & 13,52 & 111,86 & 1,364 & 13,87 & 129,67 & 1,581 \\
\hline
\end{tabular}

Idade técnica de corte.

*Idade de desbaste dos índices de local $25,{ }^{* *}$ Idade de desbaste dos índices de local 21 e ${ }^{* * *}$ Idade de desbaste do índice de local 17.

Definiu-se como a idade de realização do desbaste a idade referente a três meses antes da idade em que o IMM foi máximo para cada índice de local. Assim, para os índices de locais 25, 21 e 17 a idade de desbaste foi de 40, 41 e 43 meses, respectivamente. Analisaramse intensidades de redução em área basal de 35 e 50\%. A partir dos resultados, observou-se que o efeito do desbaste nas ITCs de povoamentos desbastados foi semelhante ao observado nos povoamentos não desbastados, ou seja, índices de locais maiores geraram idades técnicas de cortes menores (Quadros 2 e 3).

Ao comparar a ITC para cada índice de local, observouse que no povoamento desbastado essa idade foi atingida mais tarde do que no povoamento não-desbastado. Para o índice de local 17, a ITC sem o desbaste foi de 46 meses e com um desbaste de $35 \%$, de 60 meses, enquanto para os índices de locais 21 e 25 a ITC sem o desbaste foi 44 e 43 meses e com o desbaste de $35 \%$, de 57 e 56 meses, respectivamente (Quadro 2).

Os resultados foram semelhantes para o índice de redução de $50 \%$, no entanto as ITCs foram atingidas mais tarde do que quando o índice de redução foi menor, de $35 \%$. Para o índice de local 17, a ITC sem o desbaste foi de 46 meses e com o desbaste de $50 \%$, de 68 meses, já para os índices de locais 21 e 25 a ITC sem o desbaste foi de 44 e 43 meses e com o desbaste de $50 \%$, de 65 e 63 meses, respectivamente (Quadro 3).

Para avaliar o efeito do desbaste na produção em volume, elaborou-se o Quadro 4. Constatou-se que na idade de máximo incremento médio mensal após o desbaste (ITC) não houve diferença significativa de produção em volume ( $\mathrm{m}^{3} / \mathrm{ha}$ ) entre o povoamento desbastado e o não-desbastado, considerando as duas intensidades de redução de 35 e $50 \%$ e para os índices de locais testados. Os resultados da maioria dos estudos com desbaste concordam em afirmar que a produção total do regime desbastado frequientemente não é maior do que a do regime não-desbastado. No entanto, o volume removido de um povoamento desbastado alcança um valor de mercado maior na época da colheita, com menor custo de colheita por $\mathrm{m}^{3}$ de madeira, gerando maior rentabilidade ao projeto (CLUTTER et.al., 1983).

R. Árvore, Viçosa-MG, v.29, n.5, p.731-739, 2005 
Quadro 4 - Produção volumétrica total na ITC do híbrido Eucalyptus grandis X E.urophylla do índice de local 21, sem desbaste e com desbaste de 35 e $50 \%$ da área basal

Table 4 - Total volumetric yield at ITC for the Eucalyptus grandis X E.urophylla hybrid, for the site index 21 months, without and with thinning of $35 \%$ and $50 \%$ of the basal area

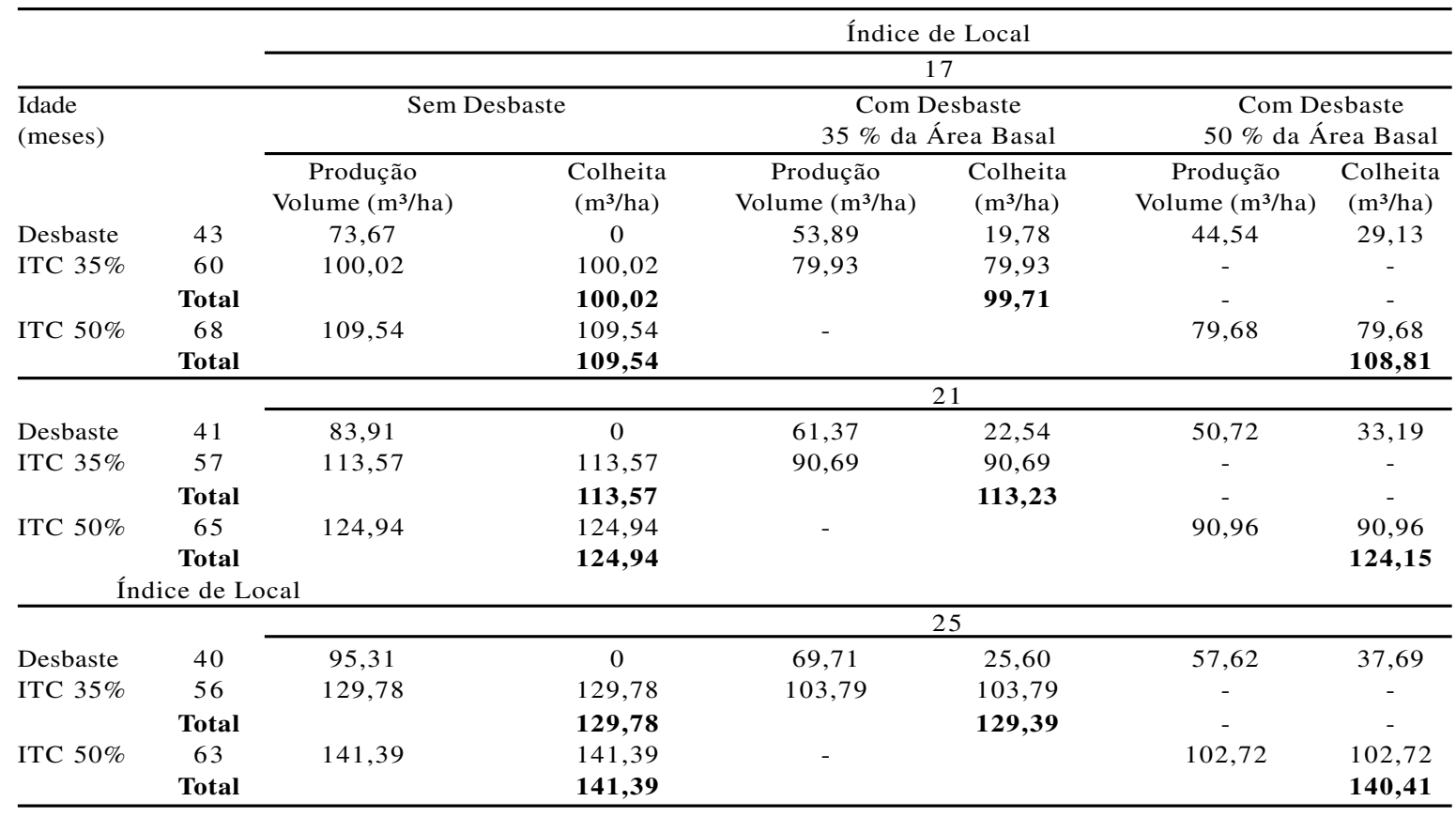

\section{CONCLUSÕES}

O modelo de Clutter atende a pressuposições estatísticas e biológicas e é eficiente para estimar a produção presente e futura em volume e área basal dos povoamentos de eucalipto submetidos a desbaste.

Observou-se, a partir dos resultados, que, à medida que são utilizados índices de local maiores na tabela de produção de densidade variável, há uma tendência de os povoamentos atingirem a idade técnica de corte (ITC) mais cedo.

Quanto maior a intensidade de desbaste, menor a idade técnica de corte.

Em termos de produção volumétrica, não houve diferença significativa do regime com desbaste e o regime sem desbaste, para os índices de local e intensidades de desbaste que foram testados.

\section{REFERÊNCIAS BIBLIOGRÁFICAS}

BUCKMAN, R.E. Growth and yield of red pine in Minnesota. Washington, D.C: USDA, 1962. 50 p. (Techical Bulletin, 1272).
GAZTELURRUTIA, M.DEL RIO.; GONZÁLES, G.M. Modelo de Simulación de claras en masas de Pinus sylvestris L. Madrid: Comunicaciones I.N.I.A., 2001. 114p. (Série monografías Forestal, 3).

CLUTTER, J.L. Compatible growth and yield models for loblolly pine. Forest Science, v.9, n.3, p.354-371, 1963.

CLUTTER, J.L. et al. Timber Management: A quantitative approach. New York: Wiley, $1983.333 \mathrm{p}$.

GUIMARÃES, D.P. Desenvolvimento de um modelo de distribuição diamétrica de passo invariante para prognose e projeção da estrutura de povoamentos de eucalipto. 1994. 160f. Tese (Doutorado em Ciência Florestal) - Universidade Federal de Viçosa, Viçosa, 1994.

HILEY, W. E. Conifers: South African methods of cultivation. London: Faber and Faber, 1959. 123 p. 
LEITE, H.G. et al. Um modelo de crescimento e produção para Pinus taeda L. na região sul do Brasil. Revista Árvore, v.25, n.1, p.105-112, 2001.

LEUSCHNER, W.A. Forest regulation, harvest scheduling and planning techniques. New York: John Wiley \& Sons., 1990. $281 \mathrm{p}$.

NOGUEIRA, G.S. et al. Determinação da idade técnica de desbaste em plantações de eucalipto utilizando o método dos ingressos percentuais. Scientia Forestalis, n.59, p-51-59, 2001.
PIENAAR, L. V.; SHIVER, B. D. An analysis and models of basal area growth in 45-year-old unthinned and thinned slash pine plantation plots. Forest Science, v. 30, n.4, p. 933-942, 1984.

STAPE, J.L.; MARTINI, E.L. Desbaste de Eucalyptus: opção de manejo para áreas com limitações ambientais ao corte raso. In: ENCONTRO TÉCNICO FLORESTAL, 5. 1991, Belo Horizonte. [Anais...] [S.L:s.n.], 1991. p.30-51.

VALDEZ-LAZALDE, J.R.; LYNCH, T.B.

Merchantable and total volume equations for thinned stands of Patula Pine In Puebla, México. Agrociência, v.34. n.6, p. 747-758, 2000. 